\title{
OPEN Dietary carbohydrates interact with $A M Y 1$ polymorphisms to influence the incidence of type 2 diabetes in Korean adults
}

\author{
Dayeon $\operatorname{Shin}^{1} \&$ Kyung Won Lee ${ }^{2 \bowtie}$
}

The relationship between $A M Y 1$ single nucleotide polymorphisms (SNPs), dietary carbohydrates, and the risk of type 2 diabetes is unclear. We aimed to evaluate this association using an ongoing largescale prospective study, namely the Korean Genome and Epidemiology Study. We selected six genetic variants of the AMY1 gene: rs10881197, rs4244372, rs6696797, rs1566154, rs1930212, and rs1999478. Baseline dietary data were obtained using a semi-quantitative food frequency questionnaire. Type 2 diabetes was defined according to the criteria of the World Health Organization and American Diabetes Association. During an average follow-up period of 12 years (651,780 person-years), 1082 out of 4552 (23.8\%) patients had type 2 diabetes. Three AMY1 SNPs were significantly associated with diabetes incidence among patients with carbohydrate intake $>65 \%$ of total energy: rs6696797, rs4244372, and rs10881197. In multivariable Cox models, Korean women with the rs6696797 AG or AA genotype had $28 \%$ higher incidence of type 2 diabetes (hazard ratio $1.28,95 \%$ confidence interval 1.06-1.55) than Korean women with the rs6696797 GG genotype. We did not observe significant associations between AMY1 SNPs, dietary carbohydrates, and diabetes incidence in Korean men. We conclude that $A M Y 1$ genetic variants and dietary carbohydrate intake influence the incidence of type 2 diabetes in Korean women only. Korean women who are minor carriers of the AMY1 rs6696797, rs4244372, and rs10881197 genotypes may benefit from a low-carbohydrate diet to prevent the future risk of type 2 diabetes.

Dietary carbohydrates may influence the development of type 2 diabetes by affecting blood glucose and insulin concentrations ${ }^{1}$. In a large prospective study of middle-aged Chinese women, rice was associated with an increased risk of type 2 diabetes $^{2}$. In a 10 -year prospective study of Japanese men, obese participants had a greater risk of type 2 diabetes when their carbohydrate intake was $>65 \%$ of the total energy intake (the acceptable macronutrient distribution range (AMDR) for carbohydrates for Japanese adults is $50-65 \%)^{3}$. In a meta-analysis of prospective cohort studies, the relative risk of type 2 diabetes was 1.11 (1.08-1.14) for each serving of white rice per day ${ }^{4}$. Multiple epidemiological studies indicate that dietary carbohydrate intake is positively associated with the risk of type 2 diabetes.

Salivary amylase is an enzyme that breaks down 1,4-alpha-glucoside bonds in oligosaccharides and polysaccharides, initiating dietary starch and glycogen digestion. Salivary amylase is encoded by the AMY1 gene, which is involved in regulating dietary starch digestion and carbohydrate metabolism. Previous research indicated that AMY1 copy number was positively associated with higher salivary amylase concentrations $\mathrm{s}^{5-8}$. Additionally, individuals with higher salivary amylase concentrations showed lower postprandial blood glucose and high insulin levels after starch consumption ${ }^{9}$. Studies have found that individuals with more salivary amylase exhibit rapid starch breakdown, leading to faster and higher blood glucose responses following starch digestion ${ }^{9}$.

Populations with high-starch diets have more AMY1 copies than populations with low-starch diets ${ }^{5}$. Starch consumption is dominant in agricultural societies, and this nutritional pressure has different effects on amylase activity ${ }^{9}$. During human evolution, the need to digest starchy food may have exerted selective pressure to increase AMY1 copy number in populations consuming high-starch diets. Korea is one of the countries where rice is a major staple ${ }^{10}$, and the calorie consumption of $57 \%$ of Korean adults exceeds the upper recommended percentage of calories from carbohydrates (65\%) from 2007 to $2012^{11}$. In recent decades, prevalence of type 2 diabetes has

${ }^{1}$ Department of Food and Nutrition, Inha University, Incheon 22212, Republic of Korea. ${ }^{2}$ Department of Home Economics Education, Korea National University of Education, Cheongju 28173, Republic of Korea. ${ }^{\square}$ email: kwlee@knue.ac.kr 


\begin{tabular}{|c|c|c|c|c|c|c|c|c|c|}
\hline \multirow[b]{2}{*}{ SNP } & \multirow[b]{2}{*}{ Chr } & \multirow[b]{2}{*}{ Minor allele } & \multirow[b]{2}{*}{ MAF } & \multicolumn{2}{|l|}{ Type 2 diabetes } & \multicolumn{2}{|l|}{ HbA1C (\%) } & \multicolumn{2}{|l|}{ Glucose (mg/dL) } \\
\hline & & & & $\mathrm{AOR}^{1}$ & $P$ value & $\beta \pm \mathrm{SE}$ & $P$ value & $\beta \pm \mathrm{SE}$ & $P$ value \\
\hline rs6696797 & 1 & A & 0.370 & $1.09(0.99-1.20)$ & 0.089 & $0.014 \pm 0.008$ & 0.095 & $0.208 \pm 0.188$ & 0.269 \\
\hline $\mathrm{rs} 4244372$ & 1 & A & 0.362 & $1.08(0.98-1.19)$ & 0.144 & $0.017 \pm 0.008$ & 0.052 & $0.24 \pm 0.188$ & 0.202 \\
\hline rs10881197 & 1 & G & 0.373 & $1.08(0.98-1.19)$ & 0.133 & $0.014 \pm 0.008$ & 0.097 & $0.217 \pm 0.188$ & 0.248 \\
\hline rs1999478 & 1 & A & 0.165 & $1.06(0.93-1.21)$ & 0.367 & $0.023 \pm 0.011$ & 0.035 & $0.289 \pm 0.245$ & 0.239 \\
\hline $\mathrm{rs} 1930212$ & 1 & G & 0.200 & $1.05(0.94-1.19)$ & 0.392 & $0.004 \pm 0.01$ & 0.677 & $0.019 \pm 0.224$ & 0.934 \\
\hline rs1566154 & 1 & G & 0.279 & $1.03(0.92-1.15)$ & 0.602 & $-0.016 \pm 0.009$ & 0.082 & $-0.151 \pm 0.203$ & 0.457 \\
\hline
\end{tabular}

Table 1. SNPs included in the study. Adjusted for age and sex. $P$ value based on the additive genetic model. SNPs single nucleotide polymorphisms, Chr chromosome, MAF minor allele frequency, $S E$ standard error, $A O R$ adjusted odds ratio.

increased $^{12}$. Low variation in $A M Y 1$ copy number is correlated with high insulin resistance in healthy Korean men $^{13}$. AMY1 copy number and carbohydrate intake differentially influence the development of type 2 diabetes.

Single nucleotide polymorphisms (SNPs) near amylase genes are highly correlated with $A M Y 1$ copy number ${ }^{14}$. Thus, nonsynonymous SNPs in the AMY1 gene may be an additional genetic influence on amylase expression ${ }^{5}$. It is important to explore both SNPs and copy number variations when analyzing the genetic basis of disease risk $^{15}$. Although the link between $A M Y 1$ copy number variations and the risk of type 2 diabetes has been widely explored, few studies have examined the associations between AMY1 genetic variants, dietary carbohydrate intake, and the incidence of type 2 diabetes. Understanding how these factors interact will provide insight regarding the role of genetic polymorphisms in type 2 diabetes etiology, with implications for further research on the physiological effect of $A M Y 1$ SNPs. In particular, examining the role of $A M Y 1$ genetic polymorphisms in the digestion of dietary carbohydrates is of great importance for improving our understanding of the type 2 diabetes pathophysiology. Using a prospective cohort study, we tested the hypothesis that $A M Y 1$ genetic polymorphisms and carbohydrate intake interact to alter the incidence of type 2 diabetes.

\section{Results}

AMY1 SNPs. Descriptions of six AMY1 SNPs are presented in Table 1. AMY1 rs6696797 was marginally associated with an increased risk for type 2 diabetes [adjusted odds ratio, 1.09; 95\% confidence interval (CI) 0.99-1.20; $P$ value $=0.089$ ]. $A M Y 1$ rs1999478 was significantly associated with glycated hemoglobin $(\beta=0.023$, standard error $=0.011, P$ value $=0.035)$. $A M Y 1$ SNPs and glucose levels were not significantly related.

Participant baseline characteristics. During a follow-up period of 12 years on average, 1082 out of 4552 (23.8\%) patients developed type 2 diabetes. General characteristics of the subjects grouped by carbohydrate intake are shown in Table 2. Participants with carbohydrate intake $>65 \%$ of total energy intake were more likely to live in Ansan, were non-smokers, older, more physically engaged, and less likely to drink than participants with carbohydrate intake $\leq 65 \%$. Those with $>65 \%$ carbohydrate of total energy also had lower intake of total energy, total protein, animal protein, fat, sugar, and dietary fiber, and expectedly, had a higher intake of carbohydrates. We also found lower fasting glucose levels in participants with carbohydrate intake $>65 \%$ of total energy intake.

Relationship between AMY1 SNPs, dietary carbohydrates, and incidence of type 2 diabetes in men. Table 3 shows the adjusted HRs and corresponding 95\% CIs for the prospective association between six $A M Y 1$ SNPs and diabetes incidence by carbohydrate intake in Korean men. After adjusting for age, residence area, education level, smoking status, alcohol consumption, physical activity, BMI, and family history of diabetes, we did not observe significant associations between individual AMY1 SNPs and the incidence of type 2 diabetes in either low- or high-carbohydrate groups in men.

Relationship between AMY1 SNPs, dietary carbohydrates, and incidence of type 2 diabetes in women. Table 4 presents the adjusted HRs and corresponding 95\% CIs for associations between AMY1 SNPs and incidence of type 2 diabetes by carbohydrate intake among Korean women. Three AMY1 SNPs were significantly associated with the incidence of type 2 diabetes in women with $>65 \%$ carbohydrate intake from energy: rs6696797, rs4244372, and rs10881197. In the > 65\% group, women with the rs6696797 AG or AA genotype had a $28 \%$ higher incidence of type 2 diabetes (HR 1.28, 95\% CI 1.06-1.55) than women with the rs6696797 GG genotype. Women with the rs4244372 AT or AA genotype had a 29\% higher incidence of type 2 diabetes than those with the TT genotype (HR 1.29, 95\% CI 1.07-1.56). Women with the rs 10881197 GC or GG genotype had a $30 \%$ greater incidence of type 2 diabetes than those with the CC genotype (HR 1.30, 95\% CI 1.08-1.57).

Cumulative incidence of type 2 diabetes by AMY1 rs 10881197 genotypes and dietary carbohydrates. Figure 1 shows the Kaplan-Meier plot of the cumulative type 2 diabetes incidence, grouped by AMY1 rs 10881197 genotypes (CC vs. GC or GG) and carbohydrate intake groups ( $\leq 65 \%$ or $>65 \%$ energy). Among the carbohydrate intake $\leq 65 \%$ group, cumulative incidence of type 2 diabetes was $10 \%, 17.9 \%$, and $23.9 \%$ in 5 , 10, and 15 years, respectively, for individuals with the rs 10881197 CC genotype. For those with the rs 10881197 


\begin{tabular}{|c|c|c|c|}
\hline & $\begin{array}{l}\text { Carbohydrate intake } \leq 65 \% \text { energy } \\
(n=550)\end{array}$ & $\begin{array}{l}\text { Carbohydrate intake }>65 \% \text { energy } \\
(n=4002)\end{array}$ & $P$ value \\
\hline Age (y) & $48.8 \pm 7.6$ & $51.4 \pm 8.5$ & $<0.0001$ \\
\hline BMI $\left(\mathrm{kg} / \mathrm{m}^{2}\right)$ & $24.5 \pm 2.9$ & $24.5 \pm 3$ & 0.971 \\
\hline \multicolumn{4}{|l|}{ Residence } \\
\hline Ansung & $190(34.6)$ & $1930(48.2)$ & $<0.0001$ \\
\hline Ansan & $360(65.5)$ & $2072(51.8)$ & \\
\hline \multicolumn{4}{|l|}{ Smoking behaviors } \\
\hline None & $267(48.6)$ & 2465 (61.6) & $<0.0001$ \\
\hline Past & $106(19.3)$ & $629(15.7)$ & \\
\hline Current & $177(32.2)$ & $908(22.7)$ & \\
\hline \multicolumn{4}{|l|}{ Family history of diabetes } \\
\hline Yes & $58(10.5)$ & $424(10.6)$ & 0.972 \\
\hline No & $492(89.5)$ & $3578(89.4)$ & \\
\hline \multicolumn{3}{|l|}{ Sex } & $<0.0001$ \\
\hline Men & $337(61.3)$ & $1844(46.1)$ & \\
\hline Women & $213(38.7)$ & $2158(53.9)$ & \\
\hline Physical activity (MET-h/week) & $154.5 \pm 97.1$ & $167.8 \pm 103.3$ & 0.004 \\
\hline Alcohol consumption (g/day) & $15.2 \pm 25.1$ & $8.4 \pm 19.9$ & $<0.0001$ \\
\hline Total energy intake (kcal) & $2297.9 \pm 732$ & $1960 \pm 577.4$ & $<0.0001$ \\
\hline Protein (g/day) & $93 \pm 33.9$ & $60.4 \pm 21.2$ & $<0.0001$ \\
\hline Plant protein (g/day) & $40.7 \pm 14.7$ & $39.5 \pm 12.7$ & 0.058 \\
\hline Animal protein (g/day) & $52.3 \pm 25.6$ & $20.9 \pm 12.7$ & $<0.0001$ \\
\hline Fat (g/day) & $56.4 \pm 22$ & $26.6 \pm 13$ & $<0.0001$ \\
\hline Carbohydrate (g/day) & $348 \pm 111.2$ & $362.3 \pm 106.6$ & 0.004 \\
\hline Sugar (g/day) & $48.3 \pm 25.3$ & $40 \pm 28.2$ & $<0.0001$ \\
\hline Dietary fiber (g/day) & $16.8 \pm 7.8$ & $14.8 \pm 8.7$ & $<0.0001$ \\
\hline HbAlc (\%) & $5.6 \pm 0.4$ & $5.6 \pm 0.4$ & 0.671 \\
\hline Fasting glucose $(\mathrm{mg} / \mathrm{dL})(\mathrm{n}=3987)$ & $84.1 \pm 8.9$ & $83.1 \pm 8.7$ & 0.009 \\
\hline Insulin $(\mu \mathrm{IU} / \mathrm{mL})(\mathrm{n}=3987)$ & $7.2 \pm 3.6$ & $7.5 \pm 4.5$ & 0.12 \\
\hline HOMA-IR $(\mathrm{n}=3987)$ & $1.5 \pm 0.8$ & $1.5 \pm 1$ & 0.382 \\
\hline
\end{tabular}

Table 2. General subject characteristics, stratified by carbohydrate intake. Data are presented as mean \pm standard deviation or $\mathrm{n}(\%) . P$ values are from chi-square tests for categorical variables and $\mathrm{t}$-tests for continuous variables. SD standard deviation, MET metabolic equivalent task, HOMA-IR homeostatic model assessment of insulin resistance.

GC or GG genotype, cumulative incidence of type 2 diabetes was $12 \%, 19.2 \%$, and $24.1 \%$ in 5,10 , and 15 years, respectively. Among the carbohydrate intake $>65 \%$ group, the cumulative incidence of type 2 diabetes was $8.1 \%, 16.3 \%$, and $21.9 \%$ in 5, 10, and 15 years for those with the rs 10881197 CC genotype, respectively. For the rs 10881197 GC or GG genotype, the cumulative incidence of type 2 diabetes was $9.7 \%, 18.7 \%$, and $24.9 \%$ in 5 , 10 , and 15 years, respectively. Overall, the cumulative incidence of type 2 diabetes was the highest in participants who had a carbohydrate intake $>65 \%$ and carried the rs 10881197 GC or GG genotype.

\section{Discussion}

In this average of 12-year, prospective population-based cohort study of middle-aged Korean adults, we demonstrated that carriers of minor alleles in AMY1 (rs6696797, rs4244372, and rs10881197) had significantly elevated incidence of type 2 diabetes if they were women who obtained $>65 \%$ of their total energy intake from carbohydrates. To the best of our knowledge, this is the first study to test the hypothesis that AMY1 genetic variants, coupled with higher carbohydrate intake, are associated with a higher incidence of type 2 diabetes. Notably, we only observed these significant associations in women and not in men, suggesting a potential sex-specific mechanism related to sex hormones or sex chromosome complement ${ }^{16}$. The Toronto Nutrigenomics and Health Study reported that $A M Y 1$ rs10881197 and AMY1 copy numbers were both associated with lower energy intake among 20-29-year-old Caucasian adults ${ }^{17}$. Thus, $A M Y 1$ SNPs may contribute to starch and energy intake.

Perry et al. ${ }^{5}$ reported that although $A M Y 1$ protein expression is correlated with $A M Y 1$ copy number, the latter does not fully explain a considerable degree of variation in the expression of the $A M Y 1$ protein $\left(\mathrm{R}^{2}=0.351\right)$. Therefore, other genetic factors, such as regulatory-region SNPs, could influence AMY1 expression. Both AMY1 copy number and $A M Y 1$ SNPs may explain between-individual differences in $A M Y 1$ protein expression, and accordingly, the reason for some individuals having a higher risk of developing type 2 diabetes than others. Here, we observed the greatest disease incidence in women who consumed high-carbohydrate diets and possessed the A allele of rs6696797, A allele of rs4244372, and G allele of rs10881197. In healthy, non-obese individuals, those with low amylase activity did not have increased insulin levels before glucose absorption began, whereas 


\begin{tabular}{|c|c|c|c|c|c|}
\hline \multicolumn{3}{|c|}{ Men with $\%$ energy carbohydrate intake $\leq 65 \%$} & \multicolumn{3}{|c|}{ Men with \% energy carbohydrate intake $>65 \%$} \\
\hline & HR & $(95 \% \mathrm{CI})$ & & HR & (95\% CI) \\
\hline \multicolumn{3}{|l|}{ rs6696797 } & \multicolumn{3}{|c|}{ rs6696797 } \\
\hline GG & 1.00 & & GG & 1.00 & \\
\hline $\mathrm{AG}+\mathrm{AA}$ & 1.14 & $(0.74-1.76)$ & $\mathrm{AG}+\mathrm{AA}$ & 1.08 & $(0.90-1.30)$ \\
\hline \multicolumn{3}{|l|}{ rs4244372 } & \multicolumn{3}{|l|}{ rs4244372 } \\
\hline TT & 1.00 & & TT & 1.00 & \\
\hline $\mathrm{AT}+\mathrm{AA}$ & 1.11 & $(0.72-1.70)$ & $\mathrm{AT}+\mathrm{AA}$ & 1.08 & $(0.90-1.30)$ \\
\hline \multicolumn{3}{|c|}{ rs10881197 } & \multicolumn{3}{|c|}{ rs10881197 } \\
\hline $\mathrm{CC}$ & 1.00 & & CC & 1.00 & \\
\hline $\mathrm{GC}+\mathrm{GG}$ & 1.15 & $(0.74-1.77)$ & $\mathrm{GC}+\mathrm{GG}$ & 1.07 & $(0.89-1.29)$ \\
\hline \multicolumn{3}{|l|}{ rs1999478 } & \multicolumn{3}{|l|}{ rs1999478 } \\
\hline $\mathrm{CC}$ & 1.00 & & CC & 1.00 & \\
\hline $\mathrm{AC}+\mathrm{AA}$ & 1.23 & $(0.80-1.89)$ & $\mathrm{AC}+\mathrm{AA}$ & 1.02 & $(0.84-1.24)$ \\
\hline \multicolumn{3}{|l|}{ rs1930212 } & \multicolumn{3}{|l|}{ rs1930212 } \\
\hline $\mathrm{AA}$ & 1.00 & & AA & 1.00 & \\
\hline $\mathrm{GA}+\mathrm{GG}$ & 0.92 & $(0.60-1.43)$ & $\mathrm{GA}+\mathrm{GG}$ & 1.02 & $(0.85-1.23)$ \\
\hline \multicolumn{3}{|l|}{ rs1566154 } & \multicolumn{3}{|l|}{ rs1566154 } \\
\hline AA & 1.00 & & AA & 1.00 & \\
\hline $\mathrm{GA}+\mathrm{GG}$ & 0.76 & $(0.50-1.16)$ & $\mathrm{GA}+\mathrm{GG}$ & 1.06 & $(0.88-1.26)$ \\
\hline
\end{tabular}

Table 3. Adjusted hazard ratios (and 95\% CIs) for associations between AMY1 SNPs and incidence of type 2 diabetes, stratified by carbohydrate intake in Korean men. Adjusted for age (years), area of residence (Ansan or Ansung), education level [elementary school or lower ( $<7$ years completed), middle/high school (7-12 years), college or higher (>12 years)], smoking status (never, former smoker, current smoker), alcohol consumption (g/day), physical activity [metabolic equivalent task (MET)-h/week], body mass index $\left(\mathrm{BMI} ; \mathrm{kg} / \mathrm{m}^{2}\right)$, and family history of diabetes (self-reports: yes, no). HR hazard ratio, CI confidence intervals.

\begin{tabular}{|c|c|c|c|c|c|}
\hline \multicolumn{3}{|c|}{ Women with $\%$ energy carbohydrate intake $\leq 65 \%$} & \multicolumn{3}{|c|}{ Women with \% energy carbohydrate intake $>65 \%$} \\
\hline & HR & $(95 \% \mathrm{CI})$ & & HR & $(95 \% \mathrm{CI})$ \\
\hline \multicolumn{3}{|l|}{ rs6696797 } & \multicolumn{3}{|c|}{ rs6696797 } \\
\hline GG & 1.00 & & GG & 1.00 & \\
\hline $\mathrm{AG}+\mathrm{AA}$ & 0.76 & $(0.39-1.46)$ & $\mathrm{AG}+\mathrm{AA}$ & 1.28 & $(1.06-1.55)$ \\
\hline \multicolumn{3}{|l|}{ rs4244372 } & \multicolumn{3}{|c|}{ rs4244372 } \\
\hline TT & 1.00 & & TT & 1.00 & \\
\hline $\mathrm{AT}+\mathrm{AA}$ & 0.80 & $(0.41-1.55)$ & $\mathrm{AT}+\mathrm{AA}$ & 1.29 & $(1.07-1.56)$ \\
\hline \multicolumn{3}{|c|}{ rs10881197 } & \multicolumn{3}{|c|}{ rs10881197 } \\
\hline $\mathrm{CC}$ & 1.00 & & $\mathrm{CC}$ & 1.00 & \\
\hline $\mathrm{GC}+\mathrm{GG}$ & 0.76 & $(0.39-1.46)$ & $\mathrm{GC}+\mathrm{GG}$ & 1.30 & $(1.08-1.57)$ \\
\hline \multicolumn{3}{|l|}{ rs1999478 } & \multicolumn{3}{|c|}{ rs1999478 } \\
\hline $\mathrm{CC}$ & 1.00 & & $\mathrm{CC}$ & 1.00 & \\
\hline $\mathrm{AC}+\mathrm{AA}$ & 0.69 & $(0.32-1.48)$ & $\mathrm{AC}+\mathrm{AA}$ & 1.14 & $(0.94-1.39)$ \\
\hline \multicolumn{3}{|l|}{ rs1930212 } & \multicolumn{3}{|c|}{ rs1930212 } \\
\hline $\mathrm{AA}$ & 1.00 & & AA & 1.00 & \\
\hline $\mathrm{GA}+\mathrm{GG}$ & 0.95 & $(0.49-1.85)$ & $\mathrm{GA}+\mathrm{GG}$ & 1.06 & $(0.88-1.27)$ \\
\hline \multicolumn{3}{|l|}{ rs1566154 } & \multicolumn{3}{|c|}{ rs1566154 } \\
\hline AA & 1.00 & & AA & 1.00 & \\
\hline $\mathrm{GA}+\mathrm{GG}$ & 0.81 & $(0.42-1.54)$ & $\mathrm{GA}+\mathrm{GG}$ & 1.00 & $(0.83-1.19)$ \\
\hline
\end{tabular}

Table 4. Adjusted hazard ratios (and 95\% CIs) for associations between AMY1 SNPs and incidence of type 2 diabetes, stratified by carbohydrate intake in Korean women. Adjusted for age (years), area of residence (Ansan or Ansung), education level [elementary school or lower ( $<7$ years completed), middle/high school (7-12 years), college or higher (>12 years)], smoking status (never, former smoker, current smoker), alcohol consumption (g/day), physical activity [metabolic equivalent task (MET)-h/week], body mass index (BMI; kg/ $\mathrm{m}^{2}$ ), and family history of diabetes (self-reports: yes, no). HR hazard ratio, CI confidence intervals. 

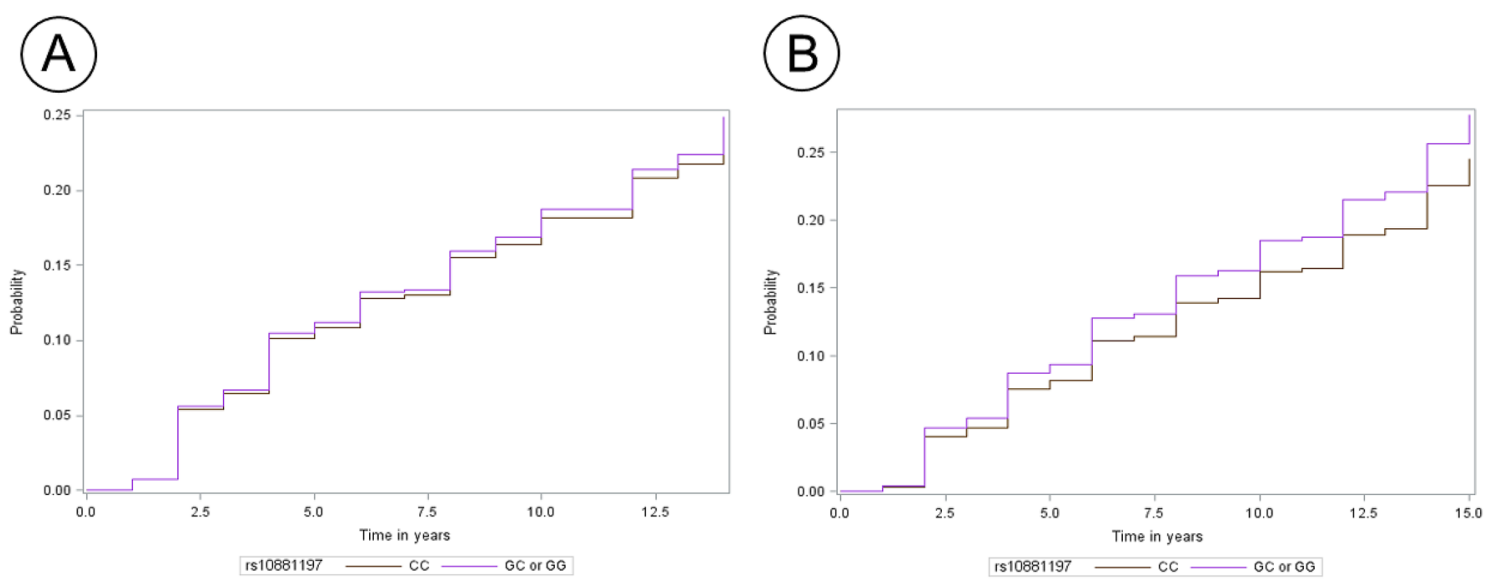

Figure 1. Cumulative incidence of type 2 diabetes by carbohydrate intake. (A) Comparison of cumulative incidence between rs 10881197 CC and rs 10881197 GC or GG genotypes for carbohydrate intake $\leq 65 \%$ of total energy intake. (B) Comparison of cumulative incidence between rs10881197 CC and rs10881197 GC or GG genotypes for those with carbohydrate intake $>65 \%$ of total energy intake.

individuals with high amylase activity had lower postprandial blood glucose concentrations ${ }^{9}$. Evolutionarily, individuals with high AMY1 copy numbers and salivary amylase activity have more rapid and efficient starch digestion mechanisms, which ensure the quick availability of blood glucose for extreme energy expenditure activities like farming or hunting 9 .

A previous study reported that high $A M Y 1$ copy number is associated with enhanced glucose absorption following an oral glucose load ${ }^{19}$. The authors suggested that the co-evolution of high $A M Y 1$ copy number with increased carbohydrate resulted in an enhanced salivary digestion of starch to maltose, followed by conversion to glucose. This enhancement optimized the efficiency of glucose absorption across the upper gastrointestinal tract. Thus, both genetic and dietary factors are implicated in the development of type 2 diabetes. Here, we found that the cumulative incidence of type 2 diabetes was highest at year 15 for participants regardless of the rs 10881197 genotypes, but significant difference in cumulative incidence by rs 10881197 genotypes (CC vs. GC or GG) was found only in the high carbohydrate-intake group. Cumulative incidence at year 15 was lowest among the participants with the rs 10881197 CC genotype and their counterparts in the low-carbohydrate-intake group. This result emphasizes the combined effects of $A M Y 1$ genotypes and dietary carbohydrates on the incidence of type 2 diabetes.

Type 2 diabetes is strongly linked with obesity ${ }^{9}$, a major cause of insulin resistance ${ }^{20,21}$. Hence, a growing body of literature has investigated the relationship between $A M Y 1$ copy number ${ }^{22-28}$ and obesity, but the outcome has been inconsistent, either failing to find any associations ${ }^{22-24}$ or identifying an inverse relationship ${ }^{25-28}$. These inconsistencies may be partially due to between-study differences in techniques for evaluating the AMY1 copy numbers and in the racial/ethnic compositions of the subject populations. Although the link between AMY1 copy numbers and obesity remains uncertain, $A M Y 1$ copy number is associated with salivary amylase concentrations ${ }^{6}$, and this relationship may contribute to individual differences in dietary starch intake. Chronically elevated blood glucose, induced by high starch intake, may cause hormonal, receptor, and physiological changes that would eventually result in type 2 diabetes $^{9}$. Furthermore, a diet rich in sugars impaired cardiac systolic and diastolic function in the mouse ${ }^{29}$. Besides SNPs in AMY1 gene, several SNPs in susceptibility genes have been associated to insulin sensitivity or insulin resistance. As genes involved in adipose tissue metabolism may influence insulin sensitivity, the polymorphisms of adiponectin gene $(-11,391 \mathrm{G}>\mathrm{A},-11,377 \mathrm{C}>\mathrm{G}$, and $+45 \mathrm{~T}>\mathrm{G})$ were associated with insulin resistance state in overweight/obese children ${ }^{18}$. It is significant to explore a wide variety of genetic factors for insulin resistance for future studies.

Our study has several limitations. First, we lacked the data to analyze salivary amylase concentration; thus, we could not further explore the linkage between AMY1 SNPs and salivary amylase concentrations. Additionally, the study was limited to participants who were over 40 years old and living in Ansan or Ansung. Therefore, our findings may not be applicable to the general Korean population.

Nevertheless, this study has several strengths. To our knowledge, it is the first to examine the association between $A M Y 1$ genetic variants and the incidence of type 2 diabetes after the stratification of the study population based on carbohydrate intake, using a 16-year prospective follow-up design. We were also able to control for multiple confounding variables because KoGES provided relevant health- and disease-related phenotypic data $^{30}$. Finally, exploring the genetic basis of type 2 diabetes risk requires measuring all forms of genetic variation, including SNPs. Accordingly, this work contributes to expanding the existing knowledge on the combined role of $A M Y 1$ and dietary carbohydrates on the development of type 2 diabetes.

In conclusion, we demonstrated that the AMY1 genetic variants and dietary carbohydrate intake influenced the incidence of type 2 diabetes among Korean women. The underlying mechanisms linking the genes, diet, and disease remain unclear, but our findings suggest that $A M Y 1$ SNPs and dietary factors are both important for type 2 diabetes etiology. This study provides a sound empirical basis for screening individuals who are minor carriers of AMY1 (rs6696797, rs4244372, and rs10881197) and have a high-carbohydrate diet, to detect future risk of type 2 diabetes. 


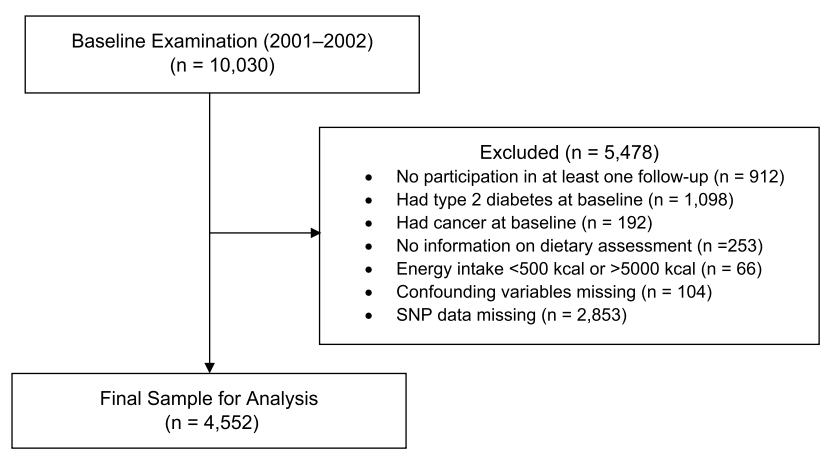

Figure 2. Flowchart of the study participants.

\section{Research design and methods}

Study design and participants. We used data from the Ansan-Ansung Cohort Study of the Korean Genome and Epidemiology Study (KoGES), an ongoing large-scale prospective study conducted by the Korea National Institute of $\mathrm{Health}^{30}$. The Ansan-Ansung study was initiated in 2001-2002 (baseline) to explore dietary and lifestyle factors that affect chronic diseases in the Korean population. It recruited 10,030 adults (40-69 years old) who resided in Ansan (urban) and Ansung (rural). Participants were followed up bi-annually, and we included their follow-up data collected until 2012.

From 10,030 participants at baseline examination (2001-2002), we excluded those who did not participate during the follow-up at least once $(n=912)$, had a diagnosis of type 2 diabetes at baseline $(n=1098)$, had a diagnosis of cancer $(n=192)$, lacked dietary information $(n=253)$, had energy intake $<500 \mathrm{kcal} /$ day or $>5000 \mathrm{kcal} /$ day $(n=66)$, had missing data for confounding variables $(n=104)$, or had no SNP data $(n=2853)$. The final analytical sample comprised 4552 individuals (Fig. 2). The KoGES study was reviewed and approved by the Institutional Review Board of the Korea Centers for Disease Control and Prevention. All participants enrolled in the study voluntarily and all gave written-informed consent. All study methods and protocols were conducted in accordance with the relevant institutional guidelines and regulations. The protocol was reviewed and approved by the Institutional Review Board of Inha University on January 31, 2020 (IRB No. 2001291A).

Dietary assessment. Dietary data were collected by well-trained interviewers at baseline, using a 103 item semi-quantitative food frequency questionnaire (FFQ). This validated FFQ was developed to assess the usual dietary intake of Korean adults who participated in the KoGES ${ }^{31}$. All participants were asked how often they consumed each food item during the previous year. They could give nine possible responses ranging from never or seldom to $\geq 3$ times per day ${ }^{31}$. To calculate the typical intake of foods and nutrients, including carbohydrates, consumption frequency for each unit of food was multiplied by the nutrient content of each food item, by referring to a nutrient database (CAN-Pro 2.0) developed by the Korean Nutrition Society ${ }^{32}$. For this study, carbohydrate intake (g/day) and percentage contribution in terms of energy (\% energy) were assessed using the FFQ.

Assessment of type 2 diabetes. During the biennial follow-up examination, type 2 diabetes was categorized as fasting blood glucose $\geq 126 \mathrm{mg} / \mathrm{dL}$ after at least $8 \mathrm{~h}$ of fasting and $2 \mathrm{~h}$ plasma glucose level of $75 \mathrm{~g}$ oral glucose tolerance test $\geq 200 \mathrm{mg} / \mathrm{dL}$, in accordance with the criteria of the World Health Organization ${ }^{33}$ and American Diabetes Association ${ }^{34}$. Other acceptable criteria were diagnosis of diabetes by a physician, use of insulin treatment, or use of diabetic medication.

Genotyping and imputation. Imputed genotypes were produced by the Korea BioBank Array Project (or KoreanChip, KCHIP) for the Korean population. Genetic data were available through the KCHIP consortium ${ }^{35}$. The KCHIP, containing about 833,535 SNPs specific to the Korean population, was designed by the Center for Genome Science, Korea National Institute of Health, Korea (4845-301, 3000-3031). Standard quality control procedures (Hardy-Weinberg equilibrium $\mathrm{P} \geq 1.0 \times 10^{-6}$, call rate $\geq 95 \%$, and INFO $\geq 0.8$ ) were used. Genetic data were imputed using SHAPEIT v2-IMPUTE v2, with the 1000 Genomes Project phase 3 reference provided by the Center for Genome Science, Korea National Institute of Health ${ }^{35}$.

Statistical analyses. Six genetic variants of $A M Y 1$ were selected: rs10881197 (minor allele, G), rs4244372 (A), rs6696797 (A), rs1566154 (G), rs1930212 (G), and rs1999478 (A) (Table 1). Genetic analysis was performed in PLINK (version 1.90 beta, https://www.cog-genomics.org/plink/1.9).

Men and women were analyzed separately. Subjects were categorized into two groups: $\leq 65 \%$ carbohydratederived energy (maximum AMDR for carbohydrates) and $>65 \%$, according to the Dietary Reference Intakes for Koreans $2020^{36}$. Baseline sociodemographic and lifestyle characteristics of participants were compared using chisquare tests for categorical variables and ANOVA for continuous variables. Hazard ratios (HRs) and 95\% confidence intervals (CIs) for the incidence of type 2 diabetes were estimated with Cox proportional hazard models, using individual AMY1 SNPs under a dominant genetic model stratified by carbohydrate intake ( $\leq 65 \%$ or $>65 \%)$. Covariates were age (years), area of residence (Ansan or Ansung), education level [elementary school or lower $(<7$ years of 
school completed), middle/high school (7-12 years), college or higher (>12 years)], smoking status (never, former smoker, current smoker), alcohol consumption (g/day), physical activity [metabolic equivalent task (MET)-h/week], body mass index (BMI; $\mathrm{kg} / \mathrm{m}^{2}$ ), and family history of diabetes (determined from self-reports: yes, no). For each AMY1 SNP, we further employed Kaplan-Meier plots to examine the cumulative incidence of type 2 diabetes during follow-up periods, stratified by carbohydrate intake ( $\leq 65 \%$ and $>65 \%)$. All statistical analyses were performed in SAS software (version 9.4; SAS Institute, Cary, NC, USA). Significance was set at $P$ value $<0.05$ (two-sided).

Received: 4 March 2021; Accepted: 30 July 2021

Published online: 18 August 2021

\section{References}

1. Meyer, K. A. et al. Carbohydrates, dietary fiber, and incident type 2 diabetes in older women. Am. J. Clin. Nutr. 71, 921-930 (2000).

2. Villegas, R. et al. Prospective study of dietary carbohydrates, glycemic index, glycemic load, and incidence of type 2 diabetes mellitus in middle-aged Chinese women. Arch. Intern. Med. 167, 2310-2316 (2007).

3. Sakurai, M. et al. Dietary carbohydrate intake, presence of obesity and the incident risk of type 2 diabetes in Japanese men. J. Diabetes Investig. 7, 343-351 (2016).

4. Hu, E. A., Pan, A., Malik, V. \& Sun, Q. White rice consumption and risk of type 2 diabetes: Meta-analysis and systematic review. BMJ 344, e1454 (2012).

5. Perry, G. H. et al. Diet and the evolution of human amylase gene copy number variation. Nat. Genet. 39, 1256-1260 (2007).

6. Mandel, A. L., Peyrot des Gachons, C., Plank, K. L., Alarcon, S. \& Breslin, P. A. Individual differences in AMY1 gene copy number, salivary a-amylase levels, and the perception of oral starch. PLoS One 5, e13352 (2010).

7. Yang, Z.-M. et al. The roles of AMY1 copies and protein expression in human salivary a-amylase activity. Physiol. Behav. 138, 173-178 (2015).

8. Alberti, G. et al. Glycemic response after starch consumption in relation to salivary amylase activity and copy-number variation of AMY1 gene. J. Food Nutr. Res. 3, 558-563 (2015).

9. Mandel, A. L. \& Breslin, P. A. S. High endogenous salivary amylase activity is associated with improved glycemic homeostasis following starch ingestion in adults. J. Nutr. 142, 853-858 (2012).

10. Chung, H.-K., Yang, H. J., Shin, D. \& Chung, K. R. Aesthetics of Korean foods: The symbol of Korean culture. J. Ethn. Foods 3, 178-188 (2016).

11. Ha, K., Kim, K., Chun, O. K., Joung, H. \& Song, Y. Differential association of dietary carbohydrate intake with metabolic syndrome in the US and Korean adults: Data from the 2007-2012 NHANES and KNHANES. Eur. J. Clin. Nutr. 72, 848-860 (2018).

12. Shin, J. Y. Trends in the prevalence and management of diabetes in Korea: 2007-2017. Epidemiol. Health 41, e2019029 (2019).

13. Choi, Y. J. et al. Association between salivary amylase (AMY1) gene copy numbers and insulin resistance in asymptomatic Korean men. Diabet. Med. 32, 1588-1595 (2015).

14. Usher, C. L. et al. Structural forms of the human amylase locus and their relationships to SNPs, haplotypes and obesity. Nat. Genet. 47, 921-925 (2015).

15. McCarroll, S. A. et al. Integrated detection and population-genetic analysis of SNPs and copy number variation. Nat. Genet. 40, 1166-1174 (2008).

16. Chella Krishnan, K., Mehrabian, M. \& Lusis, A. J. Sex differences in metabolism and cardiometabolic disorders. Curr. Opin. Lipidol. 29, 404-410 (2018).

17. Sorkin, R., Wolever, T. \& El-Sohemy, A. Genetic variation in the AMY1 gene is associated with dietary carbohydrate and starch intake in a young adult population. FASEB J. 31, 2995 (2017).

18. Petrone, A. et al. The promoter region of the adiponectin gene is a determinant in modulating insulin sensitivity in childhood obesity. Obesity (Silver Spring) 14, 1498-1504 (2006).

19. Barber, T. M. et al. AMY1 gene copy number correlates with glucose absorption and visceral fat volume, but not with insulin resistance. J. Clin. Endocrinol. Metab. 105, e3586-e3596 (2020).

20. Al-Goblan, A. S., Al-Alfi, M. A. \& Khan, M. Z. Mechanism linking diabetes mellitus and obesity. Diabetes Metab. Syndr. Obes. 7, 587-591 (2014).

21. Ahima, R. S. Connecting obesity, aging and diabetes. Nat. Med. 15, 996-997 (2009).

22. Al-Akl, N., Thompson, R. I. \& Arredouani, A. High plasma salivary a-amylase, but not high AMY1 copy number, associated with low obesity rate in Qatari adults: Cross-sectional study. Sci. Rep. 10, 17918 (2020).

23. Valsesia, A. et al. Salivary $\alpha$-amylase copy number is not associated with weight trajectories and glycemic improvements following clinical weight loss: Results from a 2-phase dietary intervention study. Am. J. Clin. Nutr. 109, 1029-1037 (2019).

24. Yong, R. Y. et al. Complex copy number variation of AMY1 does not associate with obesity in two East Asian cohorts. Hum. Mutat. 37, 669-678 (2016).

25. Falchi, M. et al. Low copy number of the salivary amylase gene predisposes to obesity. Nat. Genet. 46, 492-497 (2014).

26. Viljakainen, H. et al. Low copy number of the AMY1 locus is associated with early-onset female obesity in Finland. PLoS One 10, e0131883 (2015)

27. Marcovecchio, M. L. et al. Low AMY1 gene copy number is associated with increased body mass index in prepubertal boys. PLoS One 11, e0154961 (2016).

28. León-Mimila, P. et al. Low salivary amylase gene (AMY1) copy number is associated with obesity and gut Prevotella abundance in Mexican children and adults. Nutrients 10, 1607 (2018).

29. Carbone, S. et al. A high-sugar and high-fat diet impairs cardiac systolic and diastolic function in mice. Int. J. Cardiol. 198, 66-69 (2015).

30. Kim, Y. \& Han, B. G. Cohort profile: The Korean Genome and Epidemiology Study (KoGES) Consortium. Int. J. Epidemiol. 46, 20 (2017).

31. Ahn, Y. et al. Validation and reproducibility of food frequency questionnaire for Korean genome epidemiologic study. Eur. J. Clin. Nutr. 61, 1435-1441 (2007).

32. Korean Nutrition Society. Computer Aided Nutritional Analysis Program 4.0 for Professionals (The Korean Nutrition Society Seoul, 2011).

33. World Health Organization. Definition and Diagnosis of Diabetes Mellitus and Intermediate hyperglycaemia: Report of a WHO/IDF Consultation. (2006).

34. American Diabetes Association. Diagnosis and classification of diabetes mellitus. Diabetes Care 33, S62-S69 (2010).

35. Moon, S. et al. The Korea biobank array: Design and identification of coding variants associated with blood biochemical traits. Sci. Rep. 9, 1-11 (2019).

36. The Ministry of Health and Welfare \& The Korean Nutrition Society. Dietary Reference Intakes for Koreans 2020 (Sejong, 2020). 


\section{Acknowledgements}

This study was conducted with biosources from the National Biobank of Korea, the Centers for Disease Control and Prevention, Republic of Korea (KBN-2020-016).

\section{Author contributions}

D.S. conceptualized the study design, conducted statistical analyses, interpreted data, wrote the first draft of the manuscript, and revised the manuscript. K.W.L. conceptualized the study, conducted statistical analyses, interpreted data, supervised all aspects of implementation, and revised the manuscript. All authors read and approved the final version of the manuscript.

\section{Funding}

This work was supported by the National Research Foundation of Korea (NRF) Grant funded by the Korean government (MSIT) (Grant no. 2020R1G1A1004940).

\section{Competing interests}

The authors declare no competing interests.

\section{Additional information}

Correspondence and requests for materials should be addressed to K.W.L.

Reprints and permissions information is available at www.nature.com/reprints.

Publisher's note Springer Nature remains neutral with regard to jurisdictional claims in published maps and institutional affiliations.

(c) (i) Open Access This article is licensed under a Creative Commons Attribution 4.0 International License, which permits use, sharing, adaptation, distribution and reproduction in any medium or format, as long as you give appropriate credit to the original author(s) and the source, provide a link to the Creative Commons licence, and indicate if changes were made. The images or other third party material in this article are included in the article's Creative Commons licence, unless indicated otherwise in a credit line to the material. If material is not included in the article's Creative Commons licence and your intended use is not permitted by statutory regulation or exceeds the permitted use, you will need to obtain permission directly from the copyright holder. To view a copy of this licence, visit http://creativecommons.org/licenses/by/4.0/.

(c) The Author(s) 2021 